\title{
On stage: the reactable and other musical tangibles go real
}

\section{Sergi Jordà}

Music Technology Group, Universitat Pompeu Fabra, Ocata 1, 08003 Barcelona, Spain

E-mail: sergi.jorda@upf.edu

\begin{abstract}
This paper explores one of the application domains in which tangible and tabletop interfaces have currently shown more positive results, studying and unveiling the essential reasons that turn live music performance and tabletop interaction into promising and exiting fields of multi-disciplinary research and experimentation. The paper is structured in three parts. The first one exposes the main reasons that turn live music performance into an ideal test-bed for tangible interaction and advanced human-computer interaction. Reciprocally, the second part studies why tabletop interfaces promise remarkable new musical instruments. The third part describes the main design issues that lead to the development of the reactable, a tabletop musical instrument that has been conceived based on many of the criteria exposed on the previous two parts.
\end{abstract}

Keywords: design; interaction techniques; interfaces; musical instrument; reactable; real-time interaction; tangible tabletop interfaces.

Reference to this paper should be made as follows: Jordà, S. (2008) 'On stage: the reactable and other musical tangibles go real', Int. J. Arts and Technology, Vol. 1, Nos. 3/4, pp.268-287.

Biographical note: Sergi Jordà holds a master degree in Fundamental Physics and $\mathrm{ahD}$ in Computer Science and Digital Communication. He is a lecturer at the Pompeu Fabra University of Barcelona, where he teaches computer music, audio programming, HCI and interactive media arts. He has written many articles, books, given workshops and lectured though Europe, Asia and America. As a luthier and improviser, he likes to invent new digital musical instruments without forgetting to make music with them, and although his music has been released on various labels and he has composed for different instrumental setups and for films, he prefers the immediacy and volatility of free improvisation.

\section{Introduction}

After a seminal period that has helped laying the theoretical groundwork and the construction of descriptive taxonomies for tangible user interaction (TUI; e.g. Ishii and Ullmer, 1997; Ullmer and Ishii, 2001; Ullmer, 2002; Fishkin, 2004), many researchers agree that it is now time for posing and asking ourselves perhaps more prosaic and empirical but truly relevant questions that may help us to understand when and in which context, conditions, situations or areas, do tangible interfaces work better that other kinds of interfaces (e.g. graphical user interfaces, speech; Marshall et al., 2007). This paper explores one of the application domains that have shown more positive results, and 
proves that the current musical tangible and tabletop vogue is not only a consequence of Web 2.0 viral mechanisms, unveiling the essential reasons that turn live music performance and tabletop tangible interaction into promising and exciting fields of multidisciplinary research and experimentation. This study is approached from a theoretically grounded account and from an empirically based context both sustained on a life-term experience, as the author has been working on the realm of interactive computer music for more than two decades, as a researcher, an instrument-designer and as a performer and improviser (Jordà, 2002, 2005).

\section{Musical tabletops or 'why music performance constitutes a promising application domain for tangible and tabletop interaction?'}

\subsection{The tangible music vogue}

In recent years, we have seen a proliferation of tangible and tabletop interfaces for musical performance. The trend started with the millennium with projects such as the Audiopad (Patten, Recht and Ishii, 2002, 2006), Jam-o-drum (Blaine and Perkins, 2000) or SmallFish, ${ }^{1}$ but nowadays several 'musical tables' are being produced that it becomes difficult to keep track of every new proposal (Kaltenbrunner, 2008). Above all, the reactable has recently accomplished unparalleled mass popularity if we consider its academic origin. With demos watched millions of times on YouTube and having reached the 'real world' (and the rock stadiums) after being hand-picked by Icelandic songstress Björk for her 2007 world tour (Driver, 2007), the reactable achievements are twofold: it has made many people aware of the existence and of the potential of tangible interfaces, and it has also turned into one of the very few new digital instruments that have successfully passed the research prototype state.

It is probably not a coincidence that the two other new musical devices, which together with the reactable constitute what could be considered as the '2007 hype novel musical instruments triumvirate' (Doherty, 2008), i.e. JazzMutant's Lemur ${ }^{2}$ and Toshio Iwai-Yamaha's Tenori-On (Nishibori and Iwai, 2006), albeit not strictly tabletops, remain definitely very close to many key TUI concepts, the Lemur even being according to Buxton (2008), the first multi-touch device to be offered as a commercial product. I believe that many reasons, some more obvious than others, turn music performance into a promising test bed for tabletop-based applications. In this section, we will study the following topics:

1 collaboration and control sharing

2 real-time, multi-dimensional, continuous interaction and interaction bandwidth; and

3 complex, skilled, expressive and explorative interaction.

\subsection{Collaborative interaction and control sharing}

The social affordances associated with tables directly encourage concepts such as 'social interaction and collaboration' (Hornecker and Buur, 2006) or 'ludic interaction' (Gaver et al., 2004). Many researchers do in fact believe that the principal value of tangible interfaces may lie in their potential for facilitating kinds of collaborative activities that 
are not possible or poorly supported by single user technologies (Marshall, Rogers and Hornecker, 2007). Recently, a research community is growing around the concept of 'shareable interfaces', a generic term that refers to technologies that are specifically designed to support groups of physically co-located and co-present to work together on and around the same content. ${ }^{3}$

Sharing data between users, e.g. in the form photo collections (e.g. Shen, Lesh and Vernier 2003; Crabtree, Rodden and Mariani, 2004), probably constitutes nowadays, together with map navigation, the most popular demo for tabletop prototypes (e.g. Microsoft Surface ${ }^{4}$ ). Music performance is also about sharing, but in an essentially different way. Although traditional musical instruments have been mostly designed for an individual use and multi-user musical instruments have been truly scarce in history (Jordà, 2005), musical performance may still be one of the more archetypical group activities, and probably one of the densest form of human communication. Cognitive scientists are addressing the perceptual aspects of interdependent group playing. Benzon (2001) defines music as 'a medium through which individual brains are coupled together in shared activity', while Bischoff, Gold and Horton (1978) point that 'to bring into play the full bandwidth of communication there seems to be no substitute, for mammals at least, than the playing of music live'.

Communication in general, and musical communication makes no exception, is definitely about sharing data (Shannon, 1948), but it is not about sharing documents or files, it is about sharing real-time, on-the-fly generated data. Collective music performance with digital systems is thus better understood as a matter of sharing control over computational actions, rather than as sharing data among users. This idea of sharing control vs. the sharing of data is strongly linked to music performance but is not exclusive to it, and tangible applications with a similar philosophy are becoming more frequent. An example is the Patcher (Fernaeus and Tholander, 2006), a set of tangible resources for children in which tangible artefacts are better understood as resources for shared activity rather than as representations of shared information. Borrowing the term from contemporary social and cognitive sciences (Fernaus, Tholander and Jonsson, 2008), do even identify a 'practice turn' (Schatzki, Knorr-Cetina and Savigny, 2001) in tangible interaction and human-computer interaction (HCI) in general, that is supposing a move from a data-centric view of interaction, to a view focusing on representational forms as resources for action. Instead of relying on the transmission and sharing of data, the action-centric perspective is looking for solutions that emphasise user control, creativity and social action with interactive tools.

No matter how influential this 'turn' may be felt in a near future, the truth is that it is hard to think on shared control when models and inspirational sources come from WIMP (Windows Icons Menus Pointers)-based single-user interactive computer applications. Much of the efforts taken until today in the field of CSCW (computer-supported cooperative work) have been in that direction, trying to convert single-user applications into multi-user collaborative applications. But sequential interaction has proved to be too inflexible for collaborative work requiring concurrent and free interactions (e.g. Stefik et al., 1987; Olson et al., 1992; Begole, Rosson and Shaffer, 1999; Sun et al., 2006). Sharing control is problematic and cumbersome in WIMP-based interaction, originally conceived for single-user applications. While synchronicity problems and inconsistencies caused by simultaneous accesses can be 'solved' and managed, this interaction model does clearly not constitute the best inspirational source for the new types of collaboration we envision tangibles can convey. 


\subsection{Real-time interaction (RTI), and continuous and multi-dimensional control}

RTI is an ill-defined concept. Whereas real-time hardware architectures, real-time operating systems, real-time generated graphics or real-time media streaming, all convey clear and reasonably univocal meanings, there seems to be no canonical definition for 'real-time interaction' and I claim that the term is too often misused. RTI is not about getting 'instantaneous' or the fastest responses to our actions, very much like real-time media streaming is neither about getting media played 'as fast as possible'. In RTI, time passes independently of the users' actions, and the perception of 'instantaneity' is not as essential as the perception of 'continuity', primarily in time (but often also in space, as we will later explain). In this time continuum, the user's actions do not need to wait for each system answer; like in a real conversation, there are no fixed turns and everyone is free to say anything at any time. Action videogames, in which enemies shoot or airplanes fly (or crash) independently of our idleness, virtual and augmented reality or interactive art installations, in which users can sense the 'real' time, passing outside of the computer, and among all, performances (e.g. musical or audiovisual, such as VJing) that are executed 'in time' and (because of the audience) without a 'pause' button, are some of the paradigmatical RTI genres.

Tangible interaction, if naturalness is sought, should also be RTI, because that is the way we interact with the real world. It is true that physical board games have rules that often require ordered turns, but as well as in the physical world we are allowed to cheat, tangibles should tolerate any unconstrained and physically possible action, at anytime, anywhere, in any order and simultaneously. WIMP and productivity applications based on discrete and constrained sequences of input events (clicks, double clicks, etc.), which permanently delimit all the work flow, arguably constitute the worst RTI candidates and the worst inspirational sources we could imagine. RTI conveys in fact two 'continuums', because not only time continuity is essential but also space continuity is a clear consequence of this interaction paradigm. Actions cannot be anymore restricted to trigger events; they must also include gestures and continuous movements, and the hypertextual paradigm based on binary actions vanishes irremediably.

Traditional music performance is also continuous and multi-dimensional: a violinist continuously controls the position of the fingers on the strings as well as the position, inclination, pressure and speed of the bow. Conceptually, digital music performance is neither more constrained (although in the digital world, simultaneity and continuity can only be perceptual illusions). A quarter of a century has already passed since the MIDI implementation became a standard among all computer musicians (International MIDI Association, 1983; Loy, 1985). Since then, computer music practice has cohabited with multi-dimensional and 'continuous' control (16 MIDI channels and hundreds of possible different 'simultaneous' control parameters with 7- or 14-bit resolutions each), with a temporal granularity and precision of very few milliseconds. Does that seem too much? Not so, if we consider how much meaningful information a skilled musician is able to generate.

\subsection{Complex and explorative interaction}

Human control bandwidth is hard to evaluate objectively, but musical instruments, which are among the most complex and sophisticated machinery humans have managed to design, construct and master, can give us some clues. Traditional monophonic 
instruments frequently have three or four degrees of control. Polyphonic ones have two or three per voice while always showing limited continuous control possibilities (Pressing, 1990; Rubine and McAvinney, 1990; Vertegaal and Eaglestone, 1996; Levitin, McAdams and Adams, 2002). The reasons for these restrictions are to be found in the physical and cognitive limitations of humans and not in the inherent properties of the instruments (Fitts, 1951; Miller, 1956; Fitts and Posner, 1967; Pressing, 1988; Cook, 2001). According to Robert Moog, a flute player is able to control amplitude with a 6-bit resolution and with a temporal resolution of about $100 \mathrm{~Hz}$, while a drummer can play with a maximum frequency of $10 \mathrm{~Hz}$ controlling three parameters and an approximate resolution of 4 bit per parameter. That gives us $600 \mathrm{bits} \mathrm{sec}^{-1}$ (without considering pitch) and $120 \mathrm{bits} \mathrm{sec}^{-1}$, respectively. Moog (2004) estimates that the maximum meaningful information a skilled musician is able to generate is about $1000 \mathrm{bits} \mathrm{sec}^{-1}$. Apart from this demanding bandwidth, a very precise temporal control over several multi-dimensional and continuous parameters, sometimes even over simultaneous parallel processes is specially required in the interaction dialogue that takes place between the performer and the instrument.

While these numbers may seem overwhelming and less related to tangible interaction than the previous topics we have covered, they bring interesting subjects to debate. HCI has mostly focused on making things more efficient, not discovering until apparently quite recently that not only efficiency is relevant, but perhaps also beauty and fun (e.g. Norman, 2004; McCarthy and Wright, 2004). HCI also devotes much effort in making task-solving simpler, often forgetting Albert Einstein's famous apocryphal quote 'everything should be made as simple as possible, but no simpler'. ${ }^{5}$ Computers were not only made for making simple things, simpler, but also for making impossible things, possible (e.g. Engelbart, 1962). When complex and rich interactive processes are oversimplified, by definition, control over them can only be degraded or lost. As Buxton (1997) points out, it seems that computer devices and tools have almost never been conceived for the skilled user in mind. The study of music performance not only opens our eyes (and our ears!) to the marvels of skilled performance; it reminds us that complex interaction can also be enormously fun and rewarding for everyone, even novices. If tangible and tabletop interfaces seem ideal for exploratory and expressive activities, allowing their users to create constructions that might not be possible in other media (Marshall, Rogers and Hornecker, 2007), tangible and tabletop interfaces should neither be scared of complexity, when such complexity is needed. This is why performing arts (Sheridan and Bryan-Kinns, 2008) and music performance in particular constitute excellent realms for deeply exploring and fully exploiting the potential of this type of interaction.

\section{Tabletop musical instruments or 'why tabletop and tangible interfaces constitute good candidates for computer music performance interfaces?'}

We have studied why live music performance constitutes an ideal test-bed for tangible interaction and advanced HCI. In this section, we will try to answer a complementary and less obvious statement: "why tangible interfaces in general and tabletop interfaces in particular would constitute particularly suitable candidates for the conception and design of powerful novel digital musical instruments?' 


\subsection{Music controllers}

Dedicated interfaces for digital music performance or music controllers, as they are commonly called, have existed for years. They started to proliferate two decades ago with the advent of MIDI, and there is an ever-growing interest in this field. As a sign of this tendency, the annual conference on New Interfaces for Musical Expression (NIME) ${ }^{6}$ started in 2001 and now gathers annually more than 200 researchers, luthiers and musicians from all over the world to share their knowledge and late-breaking work on new musical interface design, a field which depicts fundamental differences with its acoustic counterpart.

Acoustic instruments consist of an excitation source that can oscillate in different ways under the control of the performer, and a resonating system that couples the vibrations of the oscillator to the surrounding air. They are built upon the laws of mechanics, and (except for keyboard instruments) the control interface and the sound generating subsystem cannot be separated or exchanged capriciously. Digital musical instruments, on their side, can always be divided into a gestural controller (or input device) that takes the control information from the performer, and a sound or music generator that plays the role of the excitation source. The controller component can typically be a simple computer mouse, a computer keyboard, a MIDI keyboard or a MIDI fader box, but with the use of sensors and appropriate analogue to digital converters, any external signal can be converted into control messages understandable by the digital system. Changes in motion, pressure, velocity, light, gravity, skin conductivity or muscle tension, almost anything, can now become a 'music controller'. Currently available controllers can be counted by hundreds, ranging from MIDI-fied versions of traditional instruments, such as saxophones, trumpets, guitars, violins, drums, xylophones or accordions, to non-imitative controllers, such as gloves, wearables, non-contact or bioelectrical devices, to mention just a few categories (Cutler, Robair and Bean, 2000). Moreover, many cheap and widely available control devices meant for the general market, such as joysticks or graphic tablets, are also often being used by musicians (Wessel and Wright, 2002; Kessous and Arfib, 2003). These controllers can preserve traditional playing modes, permitting us to blow, strike, pluck, rub or bow our 'computers'; new traditionalists in turn, may prefer to continue clicking, double-clicking, typing, pointing, sliding, twirling or dragging and dropping them. The decision is up to everyone. With the appropriate sensors, new digital instruments can also be caressed, squeezed (Weinberg and Gan, 2001), kissed, licked, danced, hummed or sung. They can even disappear or dematerialise while responding to our movements, our muscle tension or our facial expressions (Lyons, Haehnel and Tetsutani, 2003).

With the flexibility offered by MIDI, any controller can certainly be combined with any sound and music-producing device. Still, each choice is critical. As pointed by Joel Ryan, improviser, leading researcher in the NIME field and technical director of the Dutch laboratory STEIM, 'a horizontal slider, a rotary knob, a sensor that measures the pressure under one finger, an accelerometer which can measure tilt and respond to rapid movements, a sonar or an infrared system that can detect the distance between two points, each have their idiosyncratic properties' (Ryan, 1991). Any input device can become a good or a bad choice depending on the context, the parameter to control or the performer 
who will be using it. Like how the automotive engineer chooses a steering wheel over left/right incrementing buttons, 'we should not hand a musician a butterfly net when a pitchfork is required' (Puckette and Settel, 1993). The challenge remains how to integrate and transform this apparatus into coherently designed, meaningful musical experiences with emotional depth.

\subsection{Interactive music and laptop performance}

In parallel to this new interface research bloom, the laptop is progressively reaching the point of feeling as much at home on stage as a saxophone or an electric guitar. However, the contemporary musical scene does not clearly reflect this potential convergence: most laptop performers seem hesitant to switch towards the use of new hardware controllers, as if laptop performance and the exploration of post-digital sound spaces was a dialogue conducted with mice, sliders, buttons and the metaphors of business computing (Turner, 2003). There may be some reasons for this reticence, which lie precisely in the new musical possibilities of computer-based instruments.

"The philosophical instrument is sensitive; it is at the same time the musician and the instrument ... Imagine the Harpsichord having sensitivity and memory, and tell me if it would not play back by itself the airs that you have performed upon its keys. We are instruments endowed with sensibility and memory". (Diderot, 1951, p.880).

In traditional instrumental playing, every nuance, every small control variation or modulation (e.g. a vibrato or a tremolo) has to be addressed physically by the performer. The musical computer on its side, like the philosophical instrument imagined by Denis Diderot in the eighteenth century, is a system endowed with memory and knowledge, capable of handling semi-autonomous tasks. Its performer no longer needs to control directly and permanently all the aspects of the sound production, being able instead to direct and supervise the computer processes that control these details. As a result of the potential intricacy of these ongoing processes, which can be under the instrument's sole control or under a responsibility shared by the instrument and the performer, performing music with computers often tends towards an interactive dialogue between the instrument and instrumentalist. As symbolised in Figure 1, these new types of instruments often shift the centre of the performer's attention from the lower-level details to the higher-level processes that produce these details. The musician performs control strategies instead of performing data, and the instrument leans towards more intricate responses to the performer stimuli, tending to surpass the note-to-note and the 'one gesture-one acoustic event' playing paradigms present in all traditional instruments, thus allowing musicians to work at different musical levels and forcing them to take higher level and more compositional decisions on-the-fly (Jordà, 2005). The concept of 'note', the structural backbone of Western music, becomes an option rather than a necessity, now surrounded by (macrostructural) form on one side, and (microstructural) sound on the other, and the new performer moves away from the acoustic performer and approaches the orchestra conductor or even the composer (a real-time composer though!). 
Figure 1 The traditional instrument microcontrol (left) vs. the multi-threaded instrument shared control (right), which combines micro (solid line) and macro (dotted line) controls
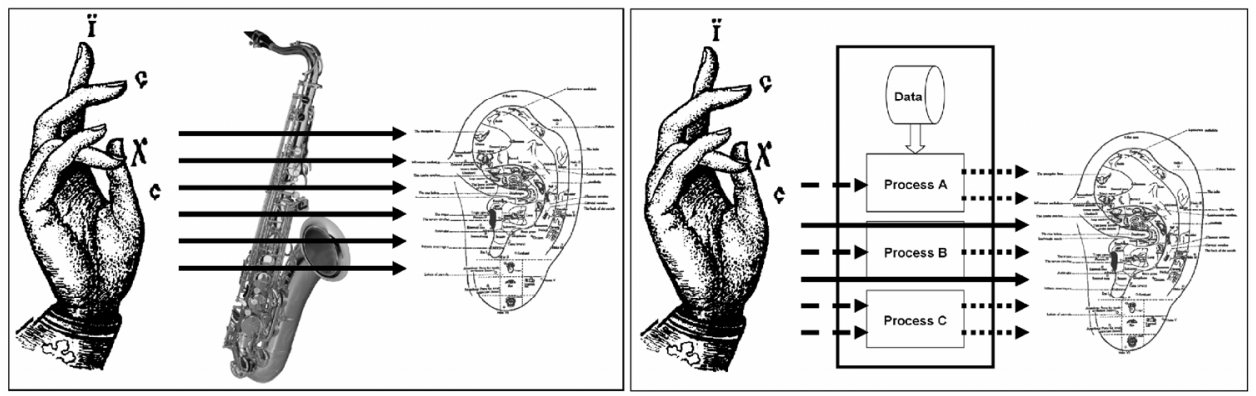

\subsection{Multi-threaded musical instruments with shared control}

Most of the music controllers currently being developed do not pursue however this 'multi-threaded and shared control' approach prolonging the traditional instrument paradigm instead. Many new musical interfaces still seem highly inspired by traditional ones, most often designed to be 'worn' and played all the time, and offering continuous, synchronous and precise control over a few dimensions. An intimate, sensitive and not necessarily highly dimensional interface of this kind (i.e. more like a violin bow, a mouthpiece or a joystick, than like a piano) will be ideally suited for direct microcontrol (i.e. sound, timbre, articulation). However, for macrostructural, indirect or higher level control, a non-wearable interface distributed and multiplexed in space and allowing intermittent access (i.e. more like a piano or a drum), and in which control can be easily and quickly transferred and recovered to/from the machine, should be undeniably preferred (Jordà, 2005). A conceptual model that recalls Marshall, Rogers and Hornecker's (2007) 'ready-at-hand vs. present-to-hand' recent discussion on the benefits of learning with tangible interfaces ('while engaged activity is important for learning so too are periods of disengaged reflection'). Tabletop interfaces, as surfaces in which objects can be alternatively grabbed or left, do certainly excel at this feature.

\subsection{Visual feedback}

In this 'shared performance' model in which performers tend to frequently delegate and shift control to the instrument, all affordable ways for monitoring ongoing processes and activities are especially welcome. Visual feedback becomes thus a significant asset for allowing these types of instruments to dynamically 'communicate' the states and the behaviours of their musical processes (Jordà, 2003). Cockpit displays may not be strictly essential for piloting a jet but I wonder how many pilots would choose to fly with their displays turned off. For the same motives, it is in fact the screen and not the mouse what laptop performers do not want to miss! And yet, not many new musical interfaces profit from the display capabilities of digital computers, which take us again to tangible interfaces.

TUIs combine control and representation within a physical artefact (Ullmer and Ishii, 2001). In table-based tangible interfaces, digital information can become graspable with the direct manipulation of simple objects that are available on a table surface. This can be 
attained by combining the tracking of control objects on the table, with projection techniques that convert the table into a flat screening surface. A table with these characteristics can favour nearly unlimited multi-parametric and shared control, interaction and exploration, multi-user collaboration, while it can also contribute to delicate and intimate interaction (e.g. moving and turning two objects with both hands). Moreover, the seamless integration of visual feedback and physical control, which eliminates the indirection component present in a conventional screen + pointer system, brings a more direct, natural, intuitive and rich interaction. I firmly believe that these interfaces can fulfil many of the special needs brought by the new live computer music performance paradigms. To conclude this section, it is also worth noting that the visual feedback brought by these interfaces, can partially solve another relevant problem of laptop music performance, such as the perception difficulties and the lack of understanding what these types of concerts provoke in the audience (Turner, 2003), which could be synthesised as 'how could we readily distinguish an artist performing with powerful generative software tools, from someone checking their e-mail whilst DJ-ing with iTunes?' (Collins, 2003). With the help of cameras and screens, new tangible musical instruments such as the Tenori-On, the Lemur or the reactable are in fact bringing the physical performance back to live computer music.

\section{The reactable}

\subsection{Design goals}

The reactable project was started in 2003 by a team of four researchers (Marcos Alonso, Günter Geiger, Martin Kaltenbrunner and Sergi Jordà) of the Music Technology Group in the Pompeu Fabra University in Barcelona (Jordà et al., 2005). The project did not set out from the idea of exploring musical applications on tabletop interfaces, but rather from our long experience as digital luthiers and computer music performers, and the objective of conceiving the best computer-based musical instrument we could imagine, without being constrained by any technological issue (Jordà, 2003). All technological problems, such as the now widely used open-source tracking library reacTIVision ${ }^{7}$ (Bencina, Kaltenbrunner and Jordà, 2005; Kaltenbrunner and Bencina, 2007), were faced and solved as the project evolved: 'we first decided what we wanted to build and then we discovered how to build it'. The foremost goal was to design an attractive, intuitive and non-intimidating musical instrument for multi-user electronic music performance, suitable for everyone to start playing from the first minute and yet capable of the more subtle and the more complex. We all know that creating can be fun, but we were not conceiving a mere sound toy, something that could become boring and predictable after a few minutes. We were committed to create a challenging, complex and endless device; capable like a traditional music instrument of rewarding effort and time spent to master it.

Within these objectives, sexiness was not a secondary issue. Considering the speed at which technology and fashion shift in our current twenty-first century, proselytising and mastery will not be attained anymore by promising to a small child (e.g. a young violinist) long-term paradise after a decade of sacrifice. This does not necessarily depict a shift from the Judaeo-Christian moral still dominant in our Western world; it only means that not many are willing to invest years of effort in order to dominate a technology that they presume will become obsolete in the coming months. If we aim for a new instrument 
to be played and mastered, it will have to be instantly engaging, capturing the musicians' imagination from the start. Additionally, concepts such as 'non-intimidation' or 'naturalness' were much more than mere captivating candies for the newcomers, because we also aspired to create a 'right-brained' computer-based musical instrument. Something which, like all traditional music instruments and unlike most computer-based systems, could especially be played 'without thinking', because musicians do not always consciously think, most often they merely act. Multi-user interaction came also as a natural choice: when shared control becomes the norm and the available control dimensionality clearly exceeds standard human control capabilities, additional sharing between several performers seems a very logical extension.

We felt that a tabletop interface could help us in fulfilling most of the previous needs, and allow us to combine what we consider the essential aspects of acoustic instruments (i.e. direct, simultaneous and fine control of several parameters, ideally using both hands) with the added potential of computer-based music tools (i.e. the possibility to share control between the performer and the instrument over simultaneous processes, and the ability to easily monitor and quickly jump between these processes). A tabletop interface could enhance control, monitoring and feedback information, and also human collaboration. With the appropriate design, it could maximise communication bandwidth in every direction (i.e. between the human performers and the computer, between the computer and the performers, between the performers themselves and, why not, between the whole system and the audience) without feeling overwhelming. As a first decision, for promoting collaboration and eliminating head position, leading voices or privileged points-of-view and control, the table was thought to be circular. The musical model came next.

\subsection{The modular synthesis metaphor}

Modular synthesis, the musical metaphor we choose to implement is a prevalent model in electronic music, which still shows an endless potential after decades of use. Modular synthesis goes back to the first sound synthesisers, both in the digital and in the analogue domains, with Robert Moog's or Donald Buchla's Voltage-controlled synthesisers from the mid-sixties (Moog, 1965). This sound synthesis and control method, based on the interconnection of sound generator and sound processor units, can also be considered as the starting point of all the visual programming environments for sound and music, which started with the Max environment in the late 1980s, and constitute nowadays one of the more flexible and widespread paradigms for interactive music making (Puckette, 2002). Traditional modular synthesis interfaces (and visual programming languages as well) are usually controlled by connecting cables or patch cords between the outputs and the inputs of different modules (see Figure 2). This approach is considered extremely flexible but not very friendly. As Davies (2001) points out commenting a museum exhibition of electronic music instruments: 'the more controls there are on an instrument, the easier it is for someone to make a few random adjustments that result in the sound disappearing, and to be unable to reverse the process. There is little point in providing an electronic instrument in a hands-on situation unless it will always produce a sound, even if many of its capabilities are thereby excluded'. And yet, we felt than when you get rid of all unnecessary complexities, this ubiquitous dataflow model can be quite easy to understand. 
Figure 2 A Robert Moog Studio-66 System modular synthesiser from 1970 (top) and details of a similar model with patch cords applied (below)
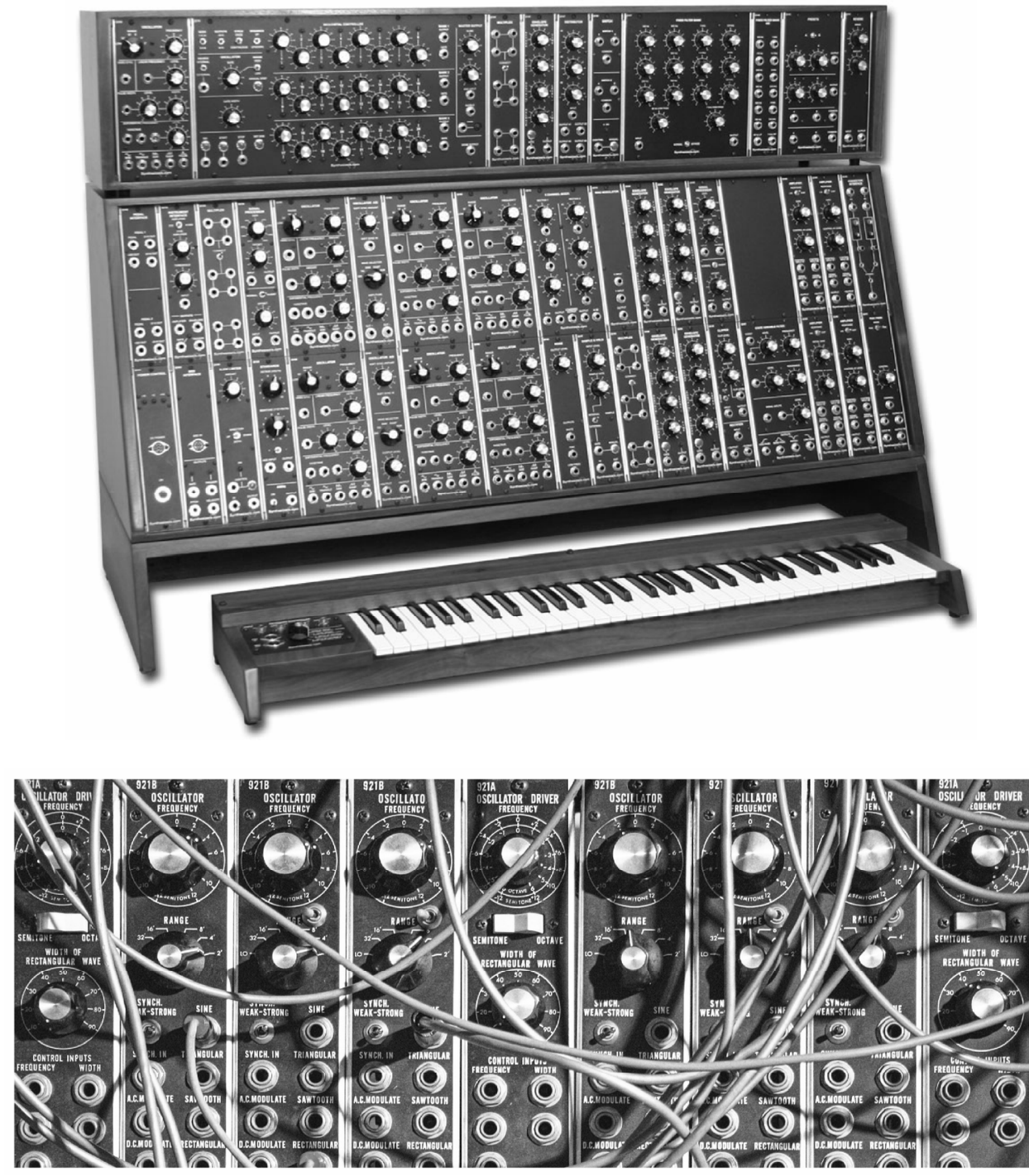

In the reactable, this modular approach is achieved by relating pucks on the table surface, where each puck has a dedicated function for the generation, modification or control of sound. Reactable's objects can be categorised into five main functional groups (see Table 1): audio generators, audio filters, controllers (which provide additional variable control to any other object), global objects (which affect the behaviour of all objects within their area of influence) and selectors (which can temporarily connect to most objects modifying some of their internal parameters). Each family is associated with a different puck shape and can have many different members, each with a distinct (humanreadable) symbol on its surface (see Figures 3, 4 and 5 and Table 1). By using tangibles, the first cognitive problem present in hardware-based modular synthesisers (and in their 
virtual replicas), which is caused by the overwhelming amount of modules, each one with its corresponding in-and-out holes and buttons, disappears; users only interact with the pucks that are used (i.e. posed on the table) at any moment. The second interaction problem, 'what to connect without going wrong', is solved in the reactable with what we call dynamic patching (Kaltenbrunner, Geiger and Jordà, 2004). Connections between the objects are managed automatically by the system without the explicit indications of the performers. This is achieved by means of a simple set of rules based on the types and affinities of the objects and on the proximity between them. As a result, only correct connections can be made. Additionally, by simply moving the pucks and bringing them into proximity with each other, performers connect and disconnect modules creating extremely dynamic synthesiser signal flow charts on-the-fly. More importantly, as shown in Figure 3, these sonic flows are permanently represented on the table surface by a graphic synthesiser that illustrates the connections between the modules by drawing a representation of the real waveforms that travel from one object to the other.

Table 1 A summary of the reactable object types

\begin{tabular}{|c|c|c|c|}
\hline & Connections & Shape & Examples \\
\hline Generators & $\begin{array}{l}\text { One audio out } \\
\mathrm{N} \text { control in }\end{array}$ & & $\begin{array}{l}\text { Square wave } \\
\text { Sampler player }\end{array}$ \\
\hline Audio filters & $\begin{array}{l}\text { One audio in } \\
\text { One audio out } \\
\mathrm{N} \text { control in }\end{array}$ & & $\begin{array}{l}\text { Resonant filter } \\
\text { Flanger }\end{array}$ \\
\hline Controllers & $\begin{array}{l}\text { One control } \\
\text { out }\end{array}$ & & $\begin{array}{l}\text { Sine wave low-frequency oscillator } \\
\text { 16-step sequencer }\end{array}$ \\
\hline Global & $\mathrm{N}$ control in & & $\begin{array}{l}\text { Metronome } \\
\text { Tonaliser }\end{array}$ \\
\hline
\end{tabular}

Figure 3 Four hands at the reactable

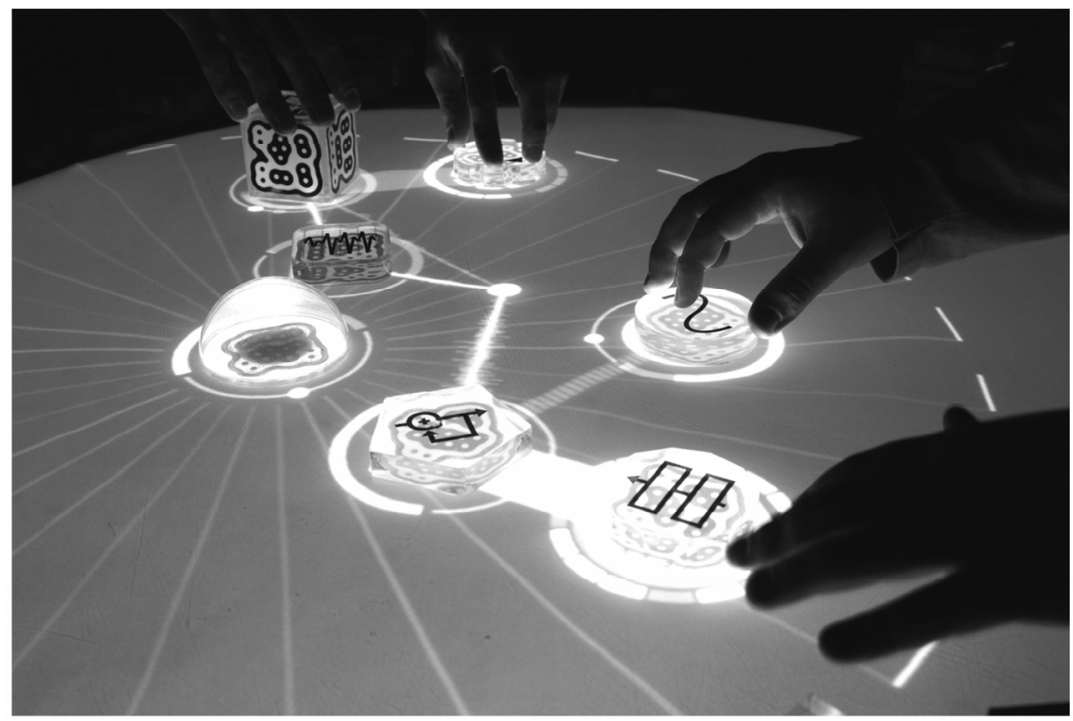


Figure 4 Modifying a parameter with a finger on the reactable

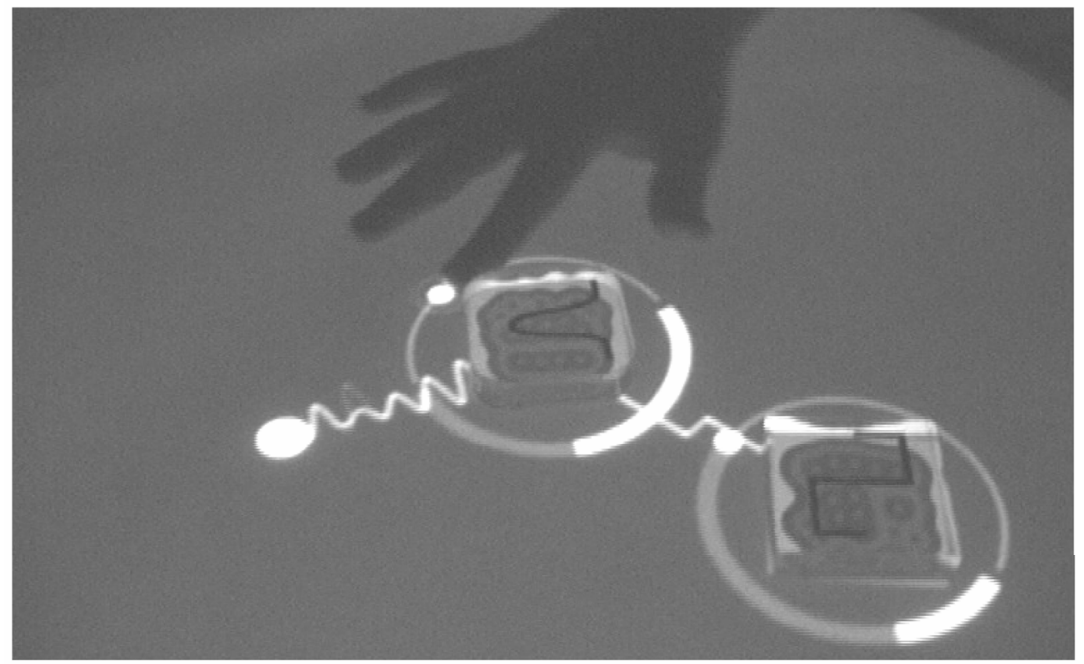

Figure 5 A snapshot showing connections between several objects. The sound generator A (a sample player) sounds alone, while the sound generator $\mathbf{B}$ (a frequency modulation synthesiser) is being controlled by the step-sequencer $\mathbf{D}$, and the resulting sound is being filtered by $\mathbf{C}$ (a resonant filter), which on its turn, is being modulated by $\mathbf{E}$ (a low-frequency sine wave oscillator). $\mathbf{G}$ is a global object (a tonaliser), which corrects the notes being generated by $\mathbf{A}$ and $\mathbf{B}$, according to the pitches that are active (dark) on its 12-note perimeter. $\mathbf{H}$ is the centre of the table, and the audio output or sink where all sounds converge

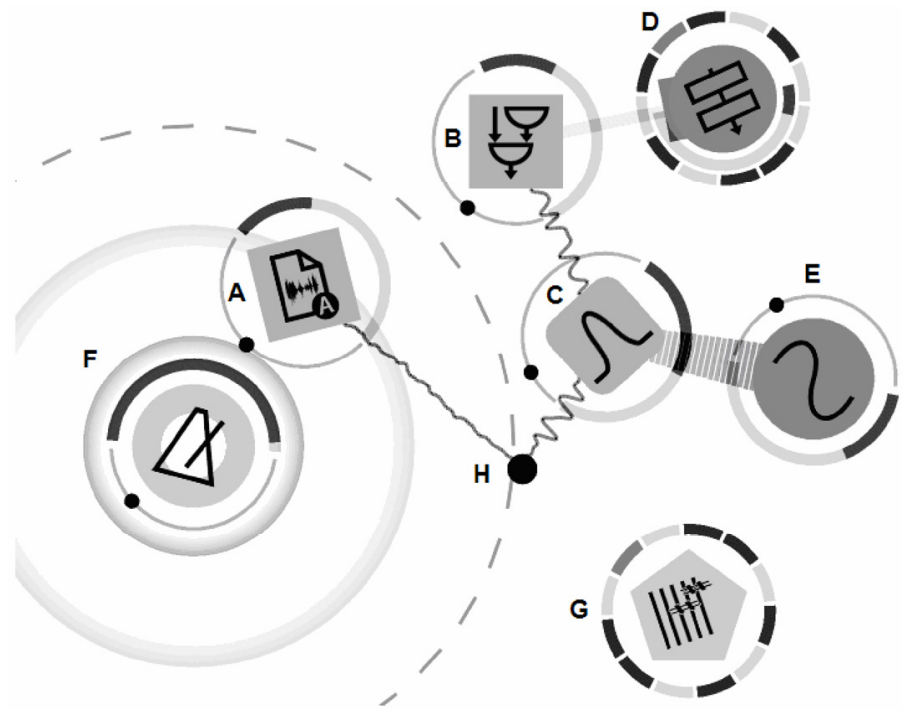




\subsection{Visual feedback and control}

Visual feedback certainly constitutes an essential component for playing the reactable and dealing with all its complexity. For permitting and easing a full control, we strictly followed three important visual design guidelines concerning the graphics projected on the table surface

1 they should permanently display all the information about the whole system state, in the simplest and more immediate way

2 they should not display anything that would not convey relevant information (i.e. no decorations)

3 this should be achieved without using any type of textual or numerical information.

As a result, only lines, dots, colours or graphical proportions evolving in time are projected on the reactable surface. The audio lines that connect the objects show the real resulting waveforms; control connections indicate the density and intensity of the values they transport; animated heartbeats reproduce the precise rates at which some 'slower' objects pulsate (the ones, such as low frequency oscillators or metronomes, which vibrate at visible rates); the graphical auras around the physical objects convey all the information about the objects' state, configuration, instant parameter values, suggesting as well the interaction possibilities they afford.

Reactable objects are synthesiser modules, each with its own control parameters, so moving them on a two-dimensional surface seemed not sufficient for fully controlling them. As a result, all reactable objects can also be spun (albeit not so popular in regular $\mathrm{HCI}$, the use of rotary knobs is a well-acquainted tradition in electronic music practice) and are also able to capture multi-touch finger interaction (see Figure 4). The use of fingers is not limited either to modifying objects' parameter. Pushing the data flow metaphor in the more natural ways, fingers can also be employed for cutting (i.e. muting) or temporarily intercepting and blocking audio connections. Visual feedback is again essential in all these cases: muted connections are represented by straight dotted lines; circular fuel gauges or discrete buttons and steps, surround many of the objects, indicating their rotational values and the possibility to click or drag with the fingers on several areas (see objects in Figure 5).

\subsection{Interacting with the reactable: lessons learned}

Since its first presentation at the Audio Engineering Society Conference in Barcelona on May 2005, the reactable has undergone a very active life outside of the laboratory. It has been exhibited in more than 100 occasions in 30 countries $^{8}$, where it has been played by dozens of thousands users, of all ages and different backgrounds (musicians, computer music and computer graphic experts; electronic music, digital art or computer games aficionados; teenagers, families with kids, etc.). While we have not undertaken any serious user studies, feedback has always been very positive, often even passionate, showing that the reactable can be very much enjoyed even without being fully understood. We have estimated that interested people start grasping its basic principles after 10 or 15 min of completely unguided and joyful interaction. Users who spend more than 10 min often become 'addicted', and come back again many times, trying to find the special moments in which the installation is empty or at least less crowded. 
In parallel to these public installations, the reactable has been featured in concerts all over Europe, America and Asia, being performed both by its creator team, and since April 2007, also by the musician and producer Damian Taylor who plays it on Björk's world tour 2007-2008, Volta. These extremely demanding situations have certainly helped turning it into a fully mature musical instrument, a fact that is not incompatible with its continual design process refinement and evolution. The instrument is used quite differently in Björk's tour and in our own concerts. Damian Taylor uses the reactable to play specific songs with quite predetermined parts, which although open to some improvisation, have to fit within the rest of the band sound and timing. For this purpose, he uses a selector object to jump from one song to another; each song consisting primarily of a predefined set of samples associated to each sample-container cube. In this setup, the timing of the reactable is also slaved/synced to the master tempo of the whole band by means of a MIDI Time Code connection.

Our concerts are much more open, often completely improvised, and the reactable, played by one to four musicians, is habitually the only instrument on stage. The stylistic range can also vary, depending on the venue, from strongly beat-oriented techno, to more experimental electronic music. In this context, all the reactable objects (about 50 pucks) are initially distributed on a non-interactive zone around the table perimeter, and can be placed on the active surface following the musicians' will. Samples and loops are also frequently loaded on the fly (using a selector object). The high complexity attainable by the whole system, with possibly 100 continuous parameters available to the performers in a crowded situation, make impossible to 'accurately' predict the results or to play the same 'exact' piece twice. Even if 'accurate' or 'exact' are just relative concepts (we all know that two performances of the same piece will never sound exactly the same, no matter who is the performer and what the instrument is), in comparative terms, the reactable is definitely less obedient than any acoustic or electric instrument, which does not mean it is uncontrollable. It does not require much training (probably a few days) to easily recognise all the objects on the set based on their shapes and their icons, to identify their functionalities and to approximately predict their behaviour when related to other objects. In our opinion, a very good balance between expressivity, richness and control has been attained. According to Ullmer (2002), the fact that most tangible platforms map interactive objects with physical world elements that have a clear geometrical representation, often poses problems with respect to the scalability of such systems, in terms of the physical objects that can be realistically manipulated on a table, and the restricted level of complexity that can be handled within these restrictions. The reactable deals reasonably well with this scalability problem: while two objects can often be sufficient for a 'solo' intervention, the level of musical complexity that can be generated by a table full of objects can easily surpass the cognitive load of several simultaneous performers.

As I mentioned in the beginning, the reactable was not the first musical tabletop. Before starting the project we were indeed influenced by Patten, Recht and Ishii's (2002) Audiopad. But nowadays, the spread of the reactable ideas together with the availability of the open-source reacTIVision software for the visual tracking of objects on a table surface, have boosted the development of related music tables, some mere reactable clones, but with many others bringing fresh and exciting ideas to the field (e.g. Bischof et al., 2008). It is too early to assess the impact that the reactable will have on a mid-term 
of such two independent fields such as tangible interaction and digital musical instruments design, but the first reactions have definitely surpassed the more optimistic previsions.

\section{Conclusions}

I have shown the implementation of a tabletop-based musical instrument, which is proving to be successful both as a musical instrument and as a tabletop application. I firmly believe that TUIs and more precisely, table-based tangible interfaces in which digital information becomes graspable with the direct manipulation of simple objects available on a table surface, can fulfil many of the special needs brought by the new live computer music performance paradigms. This type of music performance often requires the combination of intimate and sensitive control, with a more macrostructural and higher level control, which is intermittently shared, transferred and recovered between the performer(s) and the machine. Tabletop interfaces favour multi-dimensional and continuous RTI, exploration and multi-user collaboration, while they have the potential to maximise bidirectional bandwidth while also contributing to delicate and intimate interaction. Their seamless integration of visual feedback and physical control allows also for more natural and direct interaction.

I am also convinced that the deep involvement present in both expert and novice musical performance has much to bring to other interaction fields, which involve creativity in a very broad sense, understanding that creativity is present not only in the production of nice pictures or music, but specially in the process of creation or construction of anything. Creativity, related with expressiveness and freedom, can become important in any interaction process, complex enough or free enough so that the paths to a certain goal are open, or when the goal itself is open and, as in many cases of explorative learning and entertainment, when the interaction process becomes more relevant than the results. In these contexts, the openness and potential richness of tabletop interfaces coupled with the learning from complex musical interaction practice can constitute excellent departure points for new fruitful interactive experiences.

\section{Acknowledgements}

The reactable has been developed in the Music Technology Group of the Universitat Pompeu Fabra in Barcelona, by a team of four digital luthiers: Marcos Alonso, Günter Geiger, Martin Kaltenbrunner and Sergi Jordà. The reactable family is growing with the inclusion of new wonderful performers such as Carles López. The author is indebted to all of them. He is also grateful to former reactable collaborators such as Hugo Solis and Ross Bencina, who made important contributions to the computer vision component, Chris Brown who wrote and performed Teleson, the first composition for reactable, and the former interns Ignasi Casasnovas and Gerda Strobl. The author also specially thanks Xavier Serra, Director of the Music Technology Group in Barcelona for his support, Björk, Damian Taylor, Allan Pollard and all Björk's touring team, all the festival organisers who have given the chance to show the reactable in several different contexts and the thousands of users who are continuously helping in the reactable iterative refinement. 


\section{References}

Begole, J., Rosson, M. and Shaffer, C. (1999) 'Flexible collaboration transparency: supporting worker independence in replicated application sharing systems', ACM Transactions on Computer-Human Interaction (TOCHI), Vol. 6, pp.95-132.

Bencina, R., Kaltenbrunner, M. and Jordà, S. (2005) 'Improved topological fiducial tracking in the reacTIVision system', Paper presented in the Proceedings of the 2005 IEEE Computer Society Conference on Computer Vision and Pattern Recognition, San Diego, CA.

Benzon, W.L. (2001) Beethoven's Anvil: Music in Mind and Culture. New York: Basic Books.

Bischoff, J., Gold, R. and Horton, J. (1978) 'Music for an interactive network of computers', Computer Music Journal, Vol. 2, pp.24-29.

Bischof, M., Conradi, B., Lachenmaier, P., Linde, K., Meier, M., Pötzl, P. and André, E. (2008) 'XENAKIS - combining tangible interaction with probability-based musical composition', Paper presented in the Proceedings of the 2nd International Conference on Tangible and Embedded interaction TEI'08, ACM, New York, NY.

Blaine, T. and Perkis, T. (2000) 'Jam-O-Drum, a study interaction design', Paper presented in the Proceedings of ACM DIS 2000 Conference, ACM Press, NY.

Buxton, W. (1997) 'Artists and the art of the luthier', Computer Graphics: The SIGGRAPH Quarterly, Vol. 31, pp.10-11.

Buxton, W. (2008) http://www.billbuxton.com/multitouchOverview.html. Referenced April 23, 2008.

Collins, N. (2003) 'Generative music and laptop performance', Contemporary Music Review, Vol. 22, pp.67-79.

Cook, P.R. (2001) 'Principles for designing computer music controllers', Paper presented in the Proceedings of the 2001 Workshop on New Interfaces for Musical Expression, CHI 2001, Seattle, WA.

Crabtree, A., Rodden, T. and Mariani, J. (2004) 'Collaborating around collections: informing the continued development of photoware', Paper presented in the Proceedings of the 2004 ACM Conference on Computer Supported Cooperative Work (CSCW'04), Chicago, Illinois, USA, November 06-10, 2004, ACM, New York, NY, pp.396-405.

Cutler, M., Robair, G. and Bean (2000) 'Outer limits', Electronic Musician Magazine, August 2000, pp.49-72.

Davies, H. (2001) 'The preservation of electronic musical instruments', Journal of New Music Research, Vol. 30, pp.295-302.

Diderot, D. (1951) Euvres. Paris: La Pléiade, Gallimard.

Doherty, M. (2008) 'So that's what they're doing!' National Post, Chicago, Thursday, April 10, 2008. Available at: http://www.nationalpost.com/arts/story.html?id=433968. Referenced April 23, 2008.

Driver, D. (2007) 'Alan Pollard: Mechanized Mischief', Available at: http://www.apple.com/ pro/profiles/pollard. Referenced April 23, 2008.

Einstein, A. (1934) 'On the method of theoretical physics', The Herbert Spencer Lecture, delivered at Oxford (June 10, 1933); also published in Philosophy of Science, Vol. 1, (April 1934), pp.163-169.

Engelbart, D.C. (1962). Augmenting Human Intellect: A Conceptual Framework. California: Stanford Research Inst Menlo Park.

Fernaeus, Y. and Tholander, J. (2006) 'Finding design qualities in a tangible programming space', Paper presented in the Proceedings of the CHI 2006. New York, NY: ACM.

Fernaeus, Y., Tholander, J. and Jonsson, M. (2008) 'Beyond representations: towards an actioncentric perspective on tangible interaction', Int. J. Arts and Technology, this volume.

Fishkin, K.P. (2004) 'A taxonomy for and analysis of tangible interfaces', Personal and Ubiquitous Computing, Vol. 8, pp.347-358. 
Fitts, P.M. (1951) 'Engineering psychology and equipment design', in S.S. Stevens (Ed.), Handbook of Experimental Psychology. NY: Wiley, pp.1287-1340.

Fitts, P.M. and Posner, M.I. (1967) Human Performance. Belmont, CA: Brooks/Cole.

Gaver, W.W., Bowers, J., Boucher, A., Gellerson, H., Pennington, S., Schmidt, A., Steed, A., Villars, N. and Walker, B. (2004) 'The drift table: designing for ludic engagement', Paper presented in the Proceedings of the CHI'04 Extended Abstracts on Human Factors in Computing Systems. New York, NY: ACM, pp.885-900.

Hornecker, E. and Buur, J. (2006) 'Getting a grip on tangible interaction: a framework on physical space and social interaction', Paper presented in the Proceedings of the CHI 2006, New York, NY: ACM. pp.437-446.

International MIDI Association (1983) MIDI Musical Instrument Digital Interface Specification 1.0. North Hollywood: International MIDI Association.

Ishii, H. and Ullmer, B. (1997) 'Tangible bits: towards seamless interfaces between people, bits and atoms', Paper presented in the Proceedings of the CHI 1997, New York, NY: ACM, pp.22-27.

Jordà, S. (2002) 'Improvising with computers: a personal survey (1989-2001)', Journal of New Music Research, Vol. 31, pp.1-10.

Jordà, S. (2003) 'Sonigraphical instruments: from FMOL to the reactable*', Paper presented in the Proceedings of the International Conference on New Interfaces for Musical Expression (NIME03), Montreal, Canada, pp.70-76.

Jordà, S. (2005) 'Digital Lutherie: crafting musical computers for new music performance and improvisation', PhD dissertation, Universitat Pompeu Fabra, Barcelona.

Jordà, S., Kaltenbrunner, M., Geiger, G. and Bencina, R. (2005) 'The reacTable*', Paper presented in the Proceedings of the International Computer Music Conference (ICM05), San Francisco, CA.

Kaltenbrunner, M. (2008) http://www.iua.upf.es/mtg/reacTable/?related. Referenced April 23, 2008.

Kaltenbrunner, M. and Bencina, R. (2007) 'ReacTIVision: a computer-vision framework for tablebased tangible interaction', Paper presented in the Proceedings of the 1st International Conference on Tangible and Embedded Interaction, Baton Rouge, Louisiana, pp.69-74.

Kaltenbrunner, M., Geiger, G. and Jordà, S. (2004) 'Dynamic patches for live musical performance', Paper presented in the Proceedings of the International Conference on New Interfaces for Musical Expression (NIME04), Hamamatsu, Japan, pp.19-22.

Kessous, L. and Arfib, D. (2003) 'Bimanuality in alternate musical instruments', Paper presented in the Proceedings of the 2003 International Conference on New Interfaces for Musical Expression (NIME03), Montreal, Canada, pp.140-145.

Levitin D.J., Mcadams, S. and Adams, R.L. (2002) 'Control parameters for musical instruments: a foundation for new mappings of gesture to sound', Organised Sound, Vol. 7, pp.171-189.

Loy, G. (1985) 'Musicians make a standard: the MIDI phenomenon', Computer Music Journal, Vol. 9, pp.8-26.

Lyons, M.J., Haehnel, M. and Tetsutani, N. (2003) 'Designing, playing and performing with a vision-based mouth interface', Paper presented in the Proceedings of the 2003 International Conference on New Interfaces for Musical Expression NIME03, Montreal, pp.116-121.

Marshall, P., Rogers, Y. and Hornecker, E. (2007) 'Are tangible interfaces really any better than other kinds of interfaces?', Workshop on Tangible User Interfaces in Context and Theory, CHI 2007. New York, NY: ACM.

McCarthy, J. and Wright, P. (2004) 'Technology as experience', Interactions, Vol. 11, pp.42-43.

Miller, G.A. (1956) 'The magical number seven plus or minus two: some limits on our capacity for processing information', Psychological Review, Vol. 63, pp.81-97.

Moog, R. (1965) 'Voltage-controlled electronic music modules', Journal of the Audio Engineering Society, Vol. 13, pp.200-206. 
Moog, R. (2004) Keynote speech at the Conference on New Interfaces for Musical Expression (NIME04), Hamamatsu, Japan.

Nishibori, Y. and Iwai, T. (2006) 'Tenori-On', Paper presented in the Proceedings of the 6th International Conference on New Interfaces for Musical Expression (NIME06), Paris, France.

Norman, D. (2004) Emotional Design: Why We Love (Or Hate) Everyday Things. New York, NY: Basic Books.

Olson, J., Olson, G., Strorrosten, M. and Carter, M. (1992) 'How a group-editor changes the character of a design meeting as well as its outcome', Paper presented in the Proceedings of the ACM Conference on Computer-Supported Cooperative Work, pp.91-98.

Patten, J., Recht, B. and Ishii, H. (2002) 'Audiopad: a tag-based interface for musical performance', Paper presented in the Proceedings of the International Conference on New Interface for Musical Expression NIME02, Dublin, Ireland, pp.24-26.

Patten, J., Recht, B. and Ishii, H. (2006) 'Interaction techniques for musical performance with tabletop tangible interfaces', Paper presented in the Proceedings of the ACE 2006, Advances in Computer Entertainment, Hollywood, California.

Pressing, J. (1988) 'Improvisation: methods and models', in John A. Sloboda (Ed.), Generative Processes in Music. Oxford: Clarendon Press, pp.129-178.

Pressing, J. (1990) 'Cybernetic issues in interactive performance systems', Computer Music Journal, Vol. 14, pp.12-25.

Puckette, M. (2002) 'Max at 17', Computer Music Journal, Vol. 26, pp.31-43.

Puckette, M. and Settel, Z. (1993) 'Non-obvious roles for electronics in performance enhancement', Paper presented in the Proceedings of the 1993 International Computer Music Conference. International Computer Music Association, San Francisco, CA, pp.134-137.

Rubine, D. and McAvinney, P. (1990) 'Programmable finger-tracking instrument controllers', Computer Music Journal, Vol. 14, pp.26-42.

Ryan, J. (1991) 'Some remarks on musical instrument design at STEIM', Contemporary Music Review, Vol. 6, pp.3-17.

Schatzki, T., Knorr-Cetina, K.D. and Savigny, E.V. (Eds) (2001) The Practice Turn in Contemporary Theory. Routledge: London.

Shannon, C.E. (1948) 'A mathematical theory of communication', Bell System Technical Journal, Vol. 27, pp.379-423, 623-656.

Shen, C., Lesh, N. and Vernier, F. (2003) 'Personal digital historian: story sharing around the table', Interactions, Vol. 10, pp.15-22.

Sheridan, J. and Bryan-Kinns, N. (2008) 'Designing for performative tangible interaction', Int. J. Arts and Technology, this volume.

Stefik, M., Bobrow, D., Foster, G., Lanning, S. and Tatar, D. (1987) 'WYSIWIS revised: early experiences with multiuser interfaces', ACM Transactions on Office Information Systems, Vol. 5, pp.147-167.

Sun, C., Xia, S., Sun, D., Chen, D., Shen, H. and Cai, W. (2006) 'Transparent adaptation of single-user applications for multi-user real-time collaboration', ACM Transactions on Computer-Human Interaction, Vol. 13, pp.531-582.

Turner, T. (2003) 'The resonance of the cubicle: laptop performance in post-digital musics', Contemporary Music Review, Vol. 22, pp.81-92.

Ullmer, B. (2002) 'Tangible interfaces for manipulating aggregates of digital information', $P h D$ dissertation, Massachusetts Institute of Technology.

Ullmer B. and Ishii, H. (2001) 'Emerging frameworks for tangible user interfaces', in John M. Carroll (Ed.), Human-Computer Interaction in the New Millennium. Addison-Wesley, pp.579-601.

Vertegaal, R. and Eaglestone, B. (1996) 'Comparison of input devices in an ISEE direct timbre manipulation task', Interacting with Computers, Vol. 8, pp.13-30. 
Weinberg, G. and Gan, S. (2001) 'The squeezables: toward an expressive and interdependent multi-player musical instrument', Computer Music Journal, Vol. 25, pp.37-45.

Wessel, D. and Wright, M. (2002) 'Problems and prospects for intimate musical control of computers', Computer Music Journal, Vol. 26, pp.11-22.

\section{Notes}

${ }^{1}$ SmallFish: http://hosting.zkm.de/wmuench/small_fish

${ }^{2}$ http://www.jazzmutant.com/

${ }^{3}$ Shareable Interfaces Workshop, Open University, Milton Keynes, UK, 19-20 June 2007; http://mcs.open.ac.uk/pm5923/si2007/

${ }^{4}$ Microsoft Surface: $h$ ttp://www.microsoft.com/surface/videos.html

${ }^{5}$ Einstein's (1934) original quote was in fact not so simple: 'It can scarcely be denied that the supreme goal of all theory is to make the irreducible basic elements as simple and as few as possible without having to surrender the adequate representation of a single datum of experience'.

${ }^{6}$ New Interfaces for Musical Expression, NIME: http://www.nime.org

${ }^{7}$ reacTIVision: http://mtg.upf.edu/reactable/?software

${ }^{8}$ Reactable events: http://reactable.iua.upf.edu/?events 